\title{
Isotopical Band - Gap Opening in Graphene
}

\author{
V.G. Plekhanov \\ College of Computer Science, Erika Street 7a, Tallinn, 10416, Estonia
}

Copyright (C) 2016 by authors, all rights reserved. Authors agree that this article remains permanently

open access under the terms of the Creative Commons Attribution License 4.0 International License

\begin{abstract}
The new era of nanoelectronics on the graphene basis needs the creation of the semiconducting graphene. Numerous attempts to elaborate the semiconducting graphene creation technology meet several difficulties: firstly it is quite expensive; secondly it is technically difficult to produce. In the present paper the based on principle new nuclear semiconducting graphene creation technology is described. The new method is based on the electronic excitations energy renormalization by the strong (nuclear) interaction. Suggested method provides an alternative way to experimentally tune the band - gap of graphene, which would be more efficient and more controllable than other methods that are used to open band - gap in graphene. This method not only opens the isotopical band - gap in graphene but also may throw light on the massless fermion renormalization in graphene.
\end{abstract}

Keywords Isotope Effect, Graphene, Electron - Phonon Interaction, Electrodynamics, Chromodynamics, Mass Renormalization of Massless Fermion

The richness of optical and electronic properties of graphene attracts enormous interest [1]. Carbon atom is built from 6 protons, $\mathrm{A}$ neutrons and 6 electrons, where $\mathrm{A}=6$ or 7 , yield the stable isotopes ${ }^{12} \mathrm{C}$ and ${ }^{13} \mathrm{C}$, respectively, and $\mathrm{A}=8$ characterizes the radioactive isotope ${ }^{14} \mathrm{C}$. The isotope ${ }^{12} \mathrm{C}$, with nuclear spin $\mathrm{I}=0$, is the most common one in nature with $99 \%$ of all carbon atoms, whereas only $\sim 1 \%$ are ${ }^{13} \mathrm{C}$ with nuclear spin $\mathrm{I}=1 / 2$. There are only traces of ${ }^{14} \mathrm{C}\left(10^{-12}\right.$ of all carbon atoms) which transforms into nitrogen ${ }^{14} \mathrm{~N}$ by $\beta$ - decays . Although ${ }^{14} \mathrm{C}$ only occurs rarely, it is important isotope used for historical dating (see, e.g. [2]).

Carbon, one of the most basic elements in nature, still gives a lot surprises. It is found in many different forms - allotropes - from zero dimensional fullerene, one dimensional carbon nanotubes, two dimensional graphene and graphite, to three dimensional diamond (Fig. 1) - and the properties of the various carbon allotropes can vary widely [3]. For instance, diamond is the hardest material, while graphite is one of the softest: diamond is transparent to the visible part of spectrum, while graphite is opaque; diamond is an electrical insulator, while graphite is a conductor. Very important is that all these different properties originate from the same carbon atoms, simply with different arrangements of the atomic structure.

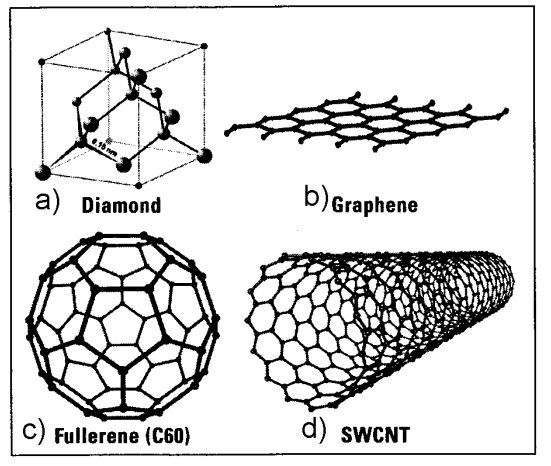

Figure 1. Structure of some representative carbon allotropes: a) diamond, b) graphene, c) fullerene, d) SWCNT.

In two-dimensional graphene, carbon atoms are periodically arranged in an infinite honeycomb lattice (Fig.1(a) in [4]). Such an atomic structure is defined by two types of bonds within the $\mathrm{sp}^{2}$ hybridization. From the four valence orbitals of the carbon atom (the $2 \mathrm{~s}, 2 \mathrm{p}_{x}, 2 \mathrm{p}_{y}$, and $2 \mathrm{p}_{z}$ orbitals, where $\mathrm{z}$ is the direction perpendicular to the sheet), the (s, $\mathrm{p}_{x}, \mathrm{p}_{y}$ ) orbitals 
combine to form the in plane $\sigma$ (bonding or occupied) and $\sigma^{*}$ (antibonding or unoccupied) orbitals. Three $\sigma$-bonds join a $\mathrm{C}$ atom to its three neighbors. They are quite strong, leading to optical - phonon frequencies much higher than observed in diamond (see below). Such orbitals are even with respect to the planar symmetry. The $\sigma$ bonds are strongly covalent bonds determining the energetic stability and the elastic properties of graphene. The remaining $\mathrm{p}_{z}$ orbital, pointing out of the graphene sheet is odd with respect to the planar symmetry and decoupled from the $\sigma$ states. From the lateral interaction with neighboring $\mathrm{p}_{z}$ orbitals (called the $\mathrm{pp} \pi$ interaction), localized $\pi$ (bonding) and $\pi^{*}$ (antibonding) orbitals are formed [5]. Graphite consists of a stack of many graphene layers. The unit cell in graphite can be primarily defined using two graphene layers translated from each other by a $\mathrm{C}$ - C distance $\left(\mathrm{a}_{c-c}=1.42 \AA\right)$. The three-dimensional structure of graphite is maintained by the weak interlayer van der Waals interaction between $\pi$ bonds of adjacent layers, which generate a weak but finite out-of-plane delocalization [1]. The bonding and antibonding $\sigma$ bands are actually strongly separated in energy $>12 \mathrm{eV}$ at , and therefore their contribution to electronic properties is commonly disregarded, while the bonding and antibonding $\pi$ states lie in the vicinity of the Fermi level (Fig. 2). The two remaining $\pi$ bands completely describe the lowenergy electronic excitations in both graphene and graphite (see [1] and references therein). The bonding $\pi$ and antibonding $\pi^{*}$ orbitals produce valence and conduction bands (Fig. 2) which cross at the charge neutrality point (Fermi level of undoped graphene) at vertices of the hexagonal Brillouin zone. Carbon atoms in a graphene plane are located at the vertices of a hexagonal lattice.

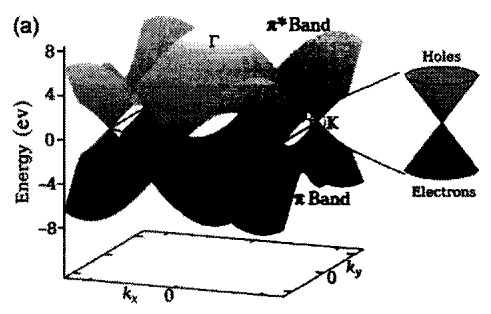

(b)

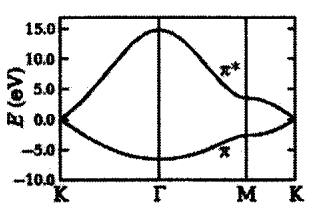

Figure 2. Energy dispersion of graphene obtained within the tight - binding approximation. a) Energy dispersion relation for graphene, drawn in the entire region of the Brillouin zone. Since in this approximation to ignore the coupling between the graphene sheets, the bands depend only on $\mathrm{k}_{x}$ and $\mathrm{k}_{y}$. The $\pi$ band is completely filled and meets the totally empty $\pi^{*}$ band at the K points. Near these points both bands linear dispersion as described in the literature. b) The dispersion along the high symmetry points $\gamma \mathrm{MK}$.

This graphene network can be regarded as a triangular Braves lattice with two atoms per unit cell (A and B). Each A- or B - type atom is surrounded by three atoms of the opposite type. In a simple neighbor model graphene is a semimetal with zero - overlap between valence and conduction bands. The energy dispersion of $\pi$ electrons in graphene was first derived in 1947 by Wallace [5] within the tight - binding approximation. In this case, the wave function of graphene is a linear combination of Bloch function for sublattice A

$$
\Phi_{A}=\frac{1}{\sqrt{\mathrm{N}}} \sum_{\overrightarrow{\mathrm{R}_{A}}} \mathrm{e}^{\mathrm{i} \overrightarrow{\mathrm{k}} \overrightarrow{\mathrm{R}_{A}}} \varphi\left(\overrightarrow{\mathrm{r}}-\overrightarrow{\mathrm{R}_{\mathrm{A}}}\right)
$$

and equilibrium function $\Phi_{B}$ for the B sublatice. Here $\mathrm{N}$ is the number of unit cells, $\overrightarrow{\mathrm{R}}_{A}$ are the position of the atom $\mathrm{A}$ and $\varphi\left(\overrightarrow{\mathrm{r}}-\overrightarrow{\mathrm{R}_{\mathrm{A}}}\right)$ is the $2 \mathrm{p}_{z}$ orbital of the atom at $\overrightarrow{\mathrm{R}}_{A}$. The sum runs over all unit cells, i.e. all possible lattice vectors. in the nearest neighbor approximation (every A site has three nearest B sites, and vice versa), the energy eigenvalues can be obtained in a closed form $[1,6]$

$$
\varepsilon\left(\mathrm{k}_{x}, \mathrm{k}_{y}\right)= \pm \gamma_{0}\left[1+4 \cos \frac{\sqrt{3} \mathrm{k}_{x} \mathrm{a}}{2} \cos \frac{\mathrm{k}_{y} \mathrm{a}}{2}+4 \cos ^{2} \frac{\mathrm{k}_{y} \mathrm{a}}{2}\right]^{1 / 2}
$$

where $\gamma_{0}$ is the transfer integral between the nearest neighbors. The energy dispersion of two - dimensional graphene according to this formula is plotted in Fig. 2(a) as a function of the wave vector $\overrightarrow{\mathrm{k}}$. The upper half of the curves is called the $\pi^{*}$ or the antibonding band while the lower one is $\pi$ or the bonding band. The two bands degenerate at the two K points given by the reciprocal space vectors $\vec{K}=(2 \pi / a)(1 / 3,1 / \sqrt{3})$ and $\vec{K}=(2 \pi / a)(-1 / 3,1 / \sqrt{3})$ points where the dispersion vanishes (see above).

Basically, graphene has redefined the limits of what a material can do: it boasts record thermal conductivity and the highest current density at room temperature ever measured (a million times that of copper!); it is the strongest material known (a hundred times stronger than steel!) yet is highly mechanically flexible; it is the least permeable material known (not even helium atoms can pass through it!); the best transparent conductive film; the thinnest material known; and the list goes on ...[6]. In the vicinity of K - points (as it can be see from Fig. 2), the low - energy electron/hole dispersion relation is proportional to momentum, rather than its square. This is analogous to the energy dispersion relation of massless relativistic electrons, so the electrons/holes of graphene are described as Dirac fermions having no mass. In a simple neighbor model graphene is a semimetal with zero - overlap between valence and conduction bands. In order to make graphene a real technology, a special issue must be solved: creating an energy gap at $\mathrm{K}$ - points in the Brillouin zone. Different attempts have been made by researches, such as patterning graphene into nanoribbon [8], forming graphene quantum dots [9 -11], making use of multilayer graphene sheets $[12,13]$ and applying an external electric field [14]. It was shown that the uniaxial strain can open a band - gap in a metallic carbon nanotubes as well as carbon nanoribbon [15]. Very shortly the isotopically band gap opening in graphene was described in [7]. The present paper is a more systematic analysis than our previous publication [7]. 
Further we will briefly discuss dependence of the electronic gap $\left(\mathrm{E}_{g}\right)$ as well as phonon states of diamond with its isotopic composition. Fig. 3 compares the edge luminescence for a natural diamond with that for a synthetic $\left({ }^{13} \mathrm{C}\right)$ diamond. The peaks labeled A, B and C due, respectively, to the recombination of a free exciton with emission of transverse - acoustic, transverse - optic and longitudinal - optic phonons having wave vector $\pm \mathrm{k}_{\min }[16,17]$.

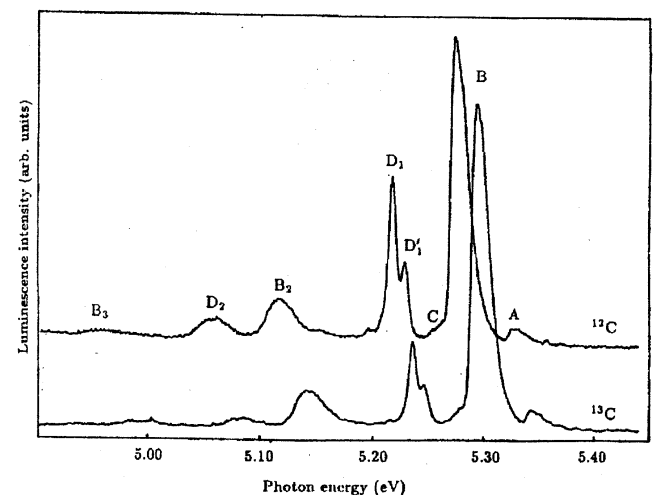

Figure 3. Cathodoluminescence spectra of ${ }^{12} \mathrm{C}$ and ${ }^{13} \mathrm{C}$ at $77 \mathrm{~K}$ [16].

As it can be seen from Fig. 3 the band gap of ${ }^{13} \mathrm{C}$ has increased by $13.6 \mathrm{meV}$. Numerous examples of band gap increasment at hard isotope substitution were collected in the papers $[16,17]$. The effect of the isotopic ${ }^{12} \mathrm{C}$ to ${ }^{13} \mathrm{C}$ ratio on the first and second - order Raman scattering of light in the diamond has been investigated in [18]. As the ${ }^{13} \mathrm{C}$ content is increased from the natural ratio $\left({ }^{12} \mathrm{C} /{ }^{13} \mathrm{C}=(1-\mathrm{x}) / \mathrm{x}\right.$, where $\left.\mathrm{x}=0.011\right)$, to the almost pure ${ }^{13} \mathrm{C}(\mathrm{x}=0.987)$, the whole spectrum has shifted towards longer wavelengths (see Fig. 4 ) in good agreement with the expected $\mathrm{M}^{-0.5}$ frequency dependence on the reduced mass $M$. For an approximately equal mix of the two isotopes, the authors reported that the features seen in the above two - phonon spectra were either broadened or unresolvable. We should stress that the main line in the first - order Raman scattering spectrum of light at $\omega=1332 \mathrm{~cm}^{-1}$ also shifts to the short-wavelength side on the $52.3 \mathrm{~cm}^{-1}[16,17]$.

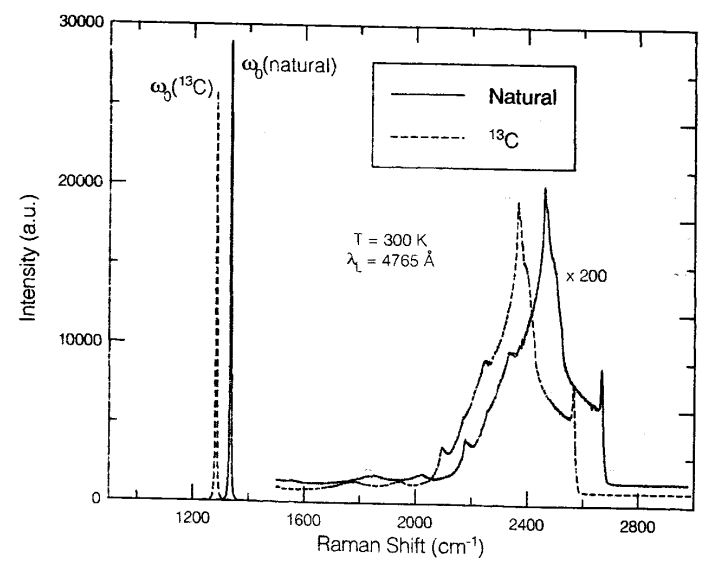

Figure 4. The Raman spectrum of a natural and a ${ }^{13} \mathrm{C}$ diamond. The spectra show the dominant first - order, Raman - active $\mathrm{F}_{2 g}$ line and the significantly weaker, quasi - continuous multi - phonon features [18].

Elastic and inelastic light scattering are powerful tools for investigating graphene [18 - 24]. Raman spectroscopy allows monitoring of doping, defects, disorder, chemical and isotope [16, 17] modifications, as well as edges and uniaxial strain. All $\mathrm{sp}^{2}$ - bonded carbons show common features in their Raman spectra, the so - called G and D peaks (see, e.g. Fig. 8 in [4]), around 1580 and $1360 \mathrm{~cm}^{-1}$ (see, e.g. [20, 21]). The $\mathrm{G}$ peak (see, also below Fig. 6) corresponds to the $\mathrm{E}_{2 g}$ phonon at the Brillouin zone center ( $\Gamma$ - point). The $\mathrm{D}$ peak is due to the breathing modes of six - atom rings and requires a defect for its activation. It comes from TO phonons around the Brillouin zone $\mathrm{K}$ point and it is activated by an intravalley scattering process [20]. The 2D peak is the second order of the D peak. This is a single peak in monolayer graphene, whereas it splits into four bands in bilayer graphene, reflecting the evolution of the band structure [4, 21]. The Raman spectrum of graphene also shows significantly less intensive defect - activated peaks such as the D' peak, which lies at $\sim 1620 \mathrm{~cm}^{-1}$. This is activated by an intravalley process i.e. connecting two points belonging to the same cone around K (see, Fig. 2) [21]. The second order of the D' peak is called 2D' peak. Since 2D and 2D' peaks originate from a Raman scattering process where momentum conservation is obtained by the participation of two phonons with opposite wave vector $(\vec{q}$ and $-\vec{q})$, they do not require the presence of defects. Thus, they are always visible in the Raman spectrum (see cited papers [18 - 24] and references therein).

Graphene is a unique material which shows properties which are not found in other materials. One of these unique features of graphene is the influence of long range strains on the electronic properties. The possibility of tuning the dynamics of carriers as well as phonons by appropriately designed strain patterns opens the way for novel applications of graphene, not possible with any other materials (see, e.g. [25] and references therein). At present time we have several reports, which have examined graphene properties under uniaxial deformation [15, 24, 25, 26]. 
Strain can be very efficiently studied by Raman spectroscopy since this modifies the crystal phonon frequency, depending on the anharmonicity of the interatomic potentials of the atoms. Raman spectra of strained graphene show significant redshifts of $2 \mathrm{D}$ and $\mathrm{G}$ band (see Table 1) because of the elongated of the carbon - carbon bonds.

Table 1. Red shift of the G and 2D bands in the Raman spectra in graphene monolayers under uniaxial tensile stress.

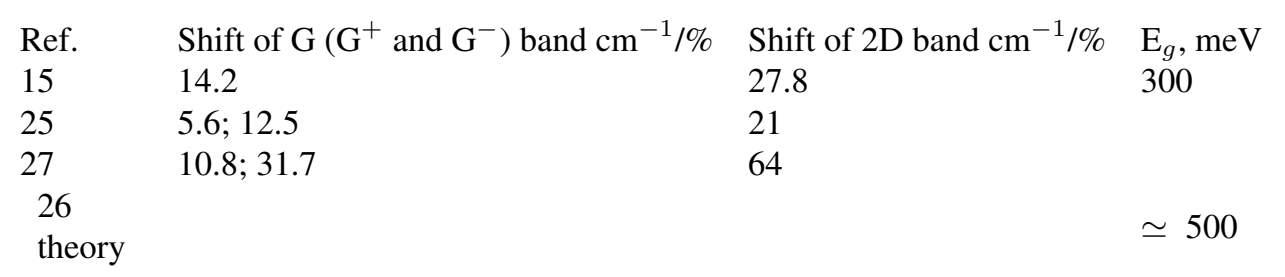

The authors of the paper [15] have proposed that by applying uniaxial strain on graphene, tunable band - gap at $\mathrm{K}$ - point can be realized. First principle calculations predicted a band - gap opening of $\simeq 300 \mathrm{meV}$ for graphene under $1 \%$ uniaxial tensile strain (Fig. 5). Thus, the strained graphene provides an alternative way to experimentally tune the band - gap of graphene, which would be more efficient and more contollable than other methods (see, above) that are used to open band gap in graphene.

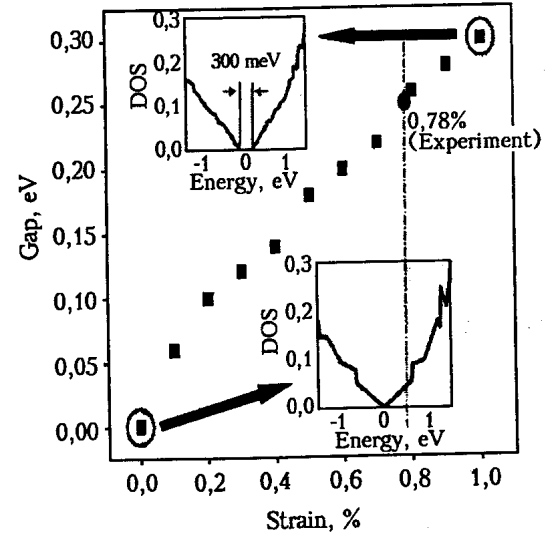

Figure 5. The bandgap of strained graphene with the increase of uniaxial tensile strain on graphene. The magnitude of gap is determined by the gap opening of density of states. The insers show the calculated density of states of unstrained and $1 \%$ tensile strained graphene. The dash line and solid dot indicate the calculated bandgap of graphene under the highest strain $(0.78 \%)$ [15].

The method of the isotope renormalization of the energy of elementary excitations in solid is often used in the last five decades and is well described in the scientific literature (see, for example reviews [16, 17]). Nowadays there is a large list of the paper devoted to the investigation of the isotope - mixed graphene [7, 11, 23, 28 - 32]. Chen and coworkers [22] have reported the first experimental study of the isotope effect on the thermal properties of graphene. The thermal conductivity $\mathrm{K}$, of isotopically pure ${ }^{12} \mathrm{C}\left(0.01{ }^{13} \mathrm{C}\right)$ graphene determined was higher than $4000 \mathrm{~W} / \mathrm{mK}$ (approximately two times more than it in diamond [14]) at the measured temperature $\mathrm{T}_{m} \sim 320 \mathrm{~K}$, and more than a factor of two higher than the value of $\mathrm{K}$ in a graphene sheets composed of a $50 \%-50 \%$ mixture of ${ }^{12} \mathrm{C}$ and ${ }^{13} \mathrm{C}$. Raman spectroscopy transferred to the $285 \mathrm{~nm} \mathrm{SiO} / \mathrm{Si}$ wafer was performed under $532 \mathrm{~nm}$ laser excitation [22]. The G peak and 2D band positions in Raman spectra of graphene with $0.01 \%, 1.1 \%, 50 \%$ and $99.2 \%{ }^{13} \mathrm{C}$ - isotope are presented in Fig. 6.

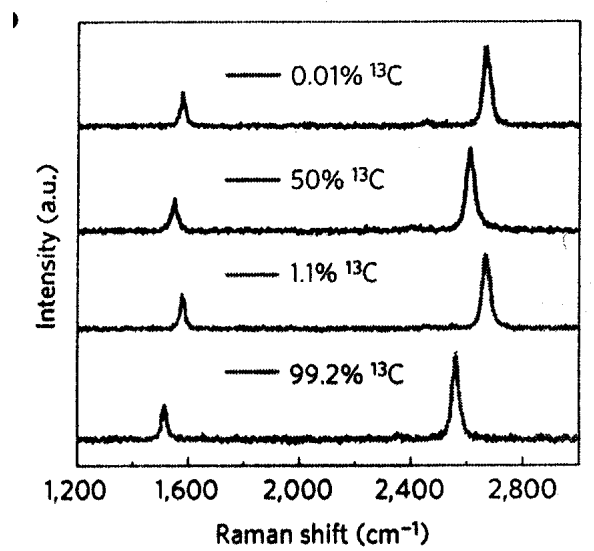

Figure 6. Raman spectra of graphena with different isotope concentration at room temperature [22]. 
Isotope shift of the $\mathrm{G}$ and 2D bands in the Raman spectra depicted on the Fig.7 [32].
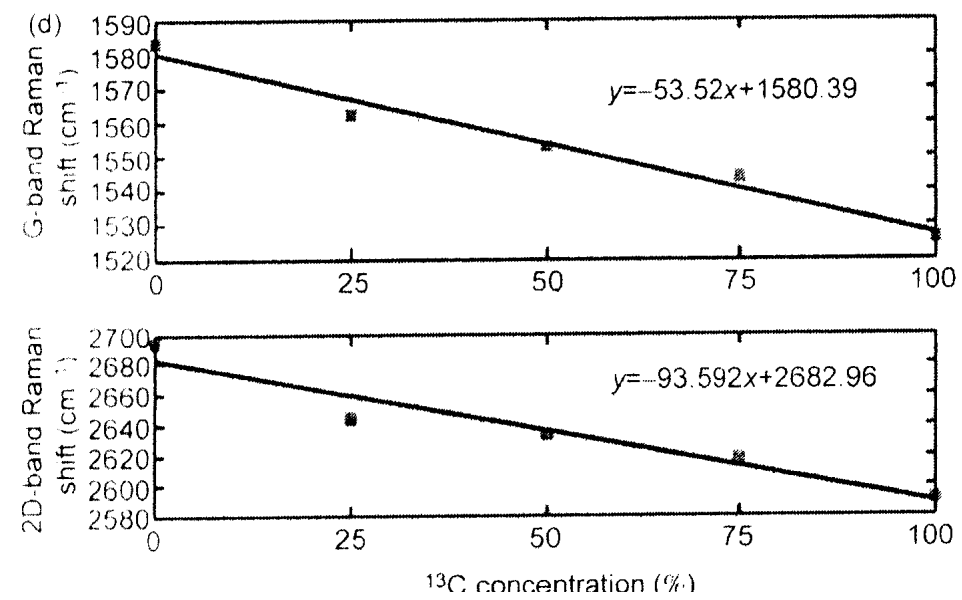

Figure 7. Peak position of $\mathrm{G}$ and $2 \mathrm{D}$ bands in Raman spectra as a function of the concentration of ${ }^{13} \mathrm{C}$ [11].

As in the case of isotope - mixed diamond $[16,17]$ the Brillouin - zone - center optical - phonon frequency $\omega$ varies with the atomic mass $\mathrm{M}$ as $\omega \sim \mathrm{M}^{-1 / 2}$ making the Raman shift for ${ }^{13} \mathrm{C}$ approximately $(12 / 13)^{-1 / 2}$ times smaller than that for ${ }^{12} \mathrm{C}$. The experimental difference between the lowest $99.2 \%{ }^{13} \mathrm{C}$ and the highest $0.01 \%{ }^{13} \mathrm{C}$ peak is $\sim 64 \mathrm{~cm}^{-1}$ which is according [22] in agreement with the theory, and attests for the high quality of isotopically modified graphene. By the way we should indicate that in the Raman spectra in diamond (with $\mathrm{sp}^{3}$ - bond) analogous shift of first - order line in Raman spectrum is equal $52.3 \mathrm{~cm}^{-1}$ [33], which is consistent with the isotope mass ratio. Substituting a light isotope $\left({ }^{12} \mathrm{C}, \mathrm{H}\right)$ with a heavy one increases the interband transition energy in the case ${ }^{12} \mathrm{C}_{x}^{13} \mathrm{C}_{1-x}$ on $14.7 \mathrm{meV}$ and $\mathrm{LiH}_{x} \mathrm{D}_{1-x}$ on $103 \mathrm{meV}$ [16]. Taking into account a more soft bond ( $\mathrm{sp}^{2}$ - bond instead $\mathrm{sp}^{3}$ - bond in diamond) isotope - induced band - gap opening in graphene of some hundreds $\mathrm{meV}$ (up to $\mathrm{E}_{g}$ of $\mathrm{Si}$ ) was predicted in paper [7]. Such estimation of the value of isotopical band - gap opening in graphene agrees with not only the results of paper [33] but with very small value $\mathrm{C}_{44}=0.5 \cdot 10^{10}$ $\mathrm{dyn} / \mathrm{cm}^{2}$. Such small value indicates on the strong electron - phonon interaction - main reason renormalization of electron excitation energy (for the details, see, e.g. [34]). Very close to isotopically renormalization of electronic excitation energy is the hydrogenation of graphene [7, 10]. In last mechanism there is observable band - gap opening in graphene. We should add that use deuteriun instead of hydrogen we may increase the value of $\mathrm{E}_{g}$ [16]. Thus, isotope substitution will be very useful method for renormalization of the band - gap in graphene - future semiconducting material. Moreover, this method allows to control not only of the strong nuclear interaction (quantum chromodynamics) but taking it into account at the renormalization of the electromagnetic interaction (quantum electrodynamics) [16]. Adding ${ }^{13} \mathrm{C}$ makes magnetic materials isotope out of graphene. Thus the use of the method of isotope effect in graphene may throw light on the renormalization of the mass of massless fermion in graphene.

\section{REFERENCES}

[1] A. Jorio, G. Dresselhaus, M.S. Dresselhaus (eds.), Carbon Nanotubes, Topics Appl. Physics 111 (Springer, Heidelberg, 2008).

[2] V.G. Plekhanov, Isotopes in Condensed Matter (Springer, Heidelberg, 2012).

[3] M.S. Dresselhaus and P.C. Eklund, Phonons in carbon nanotubes, Adv. Phys. 49, 705814 (2000).

[4] J. C. Charlier, P.C. Eklund, A.C. Ferrari, Electron and phonon properties of graphene:their relationship with carbon nanotubes, in [1] p.p.673 - 709 .

[5] P.R. Wallace, The band theory of graphite, Phys. Rev. 71, 622 - 629 (1947).

[6] S. Reich, C. Thomsen, J. Maultzsch, Carbon Nanotubes: Basic Concepts and Physics Properties (Imperial College Press, London, 2004).

[7] V.G. Plekhanov, Manifestation of the strong nuclear interactions in the isotope - induced band - gap - opening of graphene, in, Horizons in World Physics, vol. 281, 197 - 202, Nova Science, Publishers, Inc., 2013).

[8] M.Y. Han B. Ozyilmaz, Y. Zhang et al. , Energy band - gap engineering of graphene nanoribbons, Phys. Rev. Lett. 98, 206805 - 4 (2007).

[9] L.A. Ponomarenko, F. Schedin, M. Katsnelson et al., Chaotic Dirac billiard in graphene quantum dots, Science 320, 356 - 358 (2008).

[10] A. Savchenko, Transforming graphene, Science 323, 589 - 590 (2009); D.C. Elias, R.R. Nair, T.M.G. Mohiuddin et al., Control of graphene properties by reversible hydrohenation: Evidence for graphane, ibid, 323, 610 - 613 (2009). 
[11] V.G. Plekhanov, Nuclear technology creation the quantum dots in graphene, Transactions Humanitar Institute, Tallinn, 2011 , p. 66 70 (in Russian); V.G. Plekhanov, 2015 (unpublished results).

[12] See special issue Nature, June 11 (2009).

[13] K.F. Mak, C.H. Lui, T.F. Heinz, Observation of an electric - field - induced band gap in bilayer graphene by infrared spectroscopy, Phys. Rev. Lett. 102, 256405 - 4 (2009).

[14] E.V. Castro, K.S. Novoselov, S.V. Morozov, et al., Biased bilayer graphene: semiconductor with a gap tunable by the electric field effect, ibid 99, 216802 - 4 (2007).

[15] Zh.H. Ni, T. Yu, Y.H. Lu, et al., Uniaxial strain on graphene: Raman spectroscopy study and band - gap opening, ACS Nano, 3, 483 - 492 (2009).

[16] V.G. Plekhanov, Elementary excitation in isotope - mixed crystals, Phys. Reports 410, 1 -235 (2005).

[17] M. Cardona and M.L.W. Thewalt, Isotope effect on the optical spectra of semiconductors, Rev. Mod. Phys. 77, 1173 - 1224 (2005).

[18] A.K. Ramdas, and S. Rodriguez, Lattice vibrations and electronic excitations in isotopically controlled diamonds, phys. stat. sol. (b) 215, 71 - 80 (1999).

[19] S. Prawer and R.J. Nemanich, Raman spectroscopy of diamond and doped diamond, Phil. Transac. R. Soc. (London) 362, 2537 2565 (2004).

[20] M.S. Dresselhaus, G. Dresselhaus and M. Hofman, Raman spectroscopy as a probe of graphene and carbon nanotubes, ibid, 366, 231 - 236 (2008).

[21] C. Casiraghi, A. Hartschuh, H. Qian et al., Raman spectroscopy of graphene edges, Nano Lett. 9, 1433 - 1441 (2009).

[22] Sh. Chen, Q. Wu, C. Mishra et al., Thermal properties of isotopically engineered graphene, Nature Mater. 11, 203 - 207 (2012); ArXiv:cond - mat/1112.5752 (2011).

[23] A. Ferrari, Raman spectroscopy of graphene and graphite: Disorder, electron - phonon coupling, doping and nonadiabatic effects, Solid State Commun. 143, 47 - 57 (2007).

24.M. Huang, H. Yan, C. Chen et al., Raman spectroscopy of graphene under uniaxial stress: phonon softening and determination of crystallographic orientation, Proc. Natl. Acad. Sci. USA 106, 7304 - 7315 (2009).

25.M. Farjam and H. Rafii - Tabar, Comment on "Band structure engineering of graphene by strain: First - principles calculations", Phys. Rev B80, 167401 - 3 (2009).

[24] T.M. Mohiuddin, A. Lombarto, R.R. Nair et al., Uniaxial strain in graphene by Raman spectroscopy: G peak splitting, Grúneisen parameter and sample orientation, ibid, B79, 205433 - 8 (2009).

[25] N. Vandecasteele, M. Lazzeri and F. Mauri, Boosting electronic transport in carbon nanotube, Phys. Rev. Lett. 102, 196801 - 4 (2009).

[26] S.D. Costa, C. Fantini, A. Righi et al., Resonant Raman spectroscopy on enriched ${ }^{13}$ C carbon nanotubes, Carbon 49, 4919 -4723 (20011).

[27] J.F.Rodriguez - Nieva, R. Saito, S.D. Costa et al., Effect of ${ }^{13} \mathrm{C}$ doping on the optical phonon modes in graphene: Localization and Raman spectroscopy, Phys. Rev. B 85, 245406 - 8 (2012).

[28] S. Bernard, E. Whiteway, V. Yu et al., Probing the experimental phonon dispersion of graphene using ${ }^{12} \mathrm{C}$ and ${ }^{13} \mathrm{C}$ isotopes, ibid, B 86, 085409 - 5 (2012).

[29] E. del Corro, M. Kolbac, C. Fantini et al., Isotopic ${ }^{12} \mathrm{C} /{ }^{13} \mathrm{C}$ effect on the resonant Raman spectrumof twiated bilayer graphene, ibid, B 88, 155436 - 5 (2013).

[30] Z.C. Kun, L.Q. YU, T. Bo, et al., Isotope effect of the phonons mean free path in graphene by micro - Raman measurement, Science China, Phys., Mech. and Astro., 57, 1817 - 1821 (2014).

[31] K.C. Hass, M.A. Tamor, T.R. Anthony W.F. Banholzer, Lattice dynamics and Raman spectra of isotopically mixed diamons, Phys. Rev. B45, 7171 - 7182 (1992).

[32] E.G. Browman and Y.M. Kagan, Phonons in non - transition metals, in, Dynamical Properties of Solids, G.K. Horton, A.A. Maradudin (eds), Vol. 1, North - Holland Publishing Company, 1974, Chapter 4. 